\title{
Nonlinear analysis of heart rate variability in Type 2 diabetic patients
}

\author{
Mitko Gospodinov ${ }^{1 *}$, Evgeniya Gospodinova ${ }^{1}$, Ivan Domuschiev ${ }^{2}$, Nilanjan Dey ${ }^{3}$ and Amira Ashour ${ }^{4}$ \\ ${ }^{1}$ Institute of Systems Engineering and Robotics, Bulgarian Academy of Sciences, Bulgaria \\ ${ }^{2}$ II Department of Internal Diseases, Multiprofile Hospital for active treatment "St. Panteleimont”, Bulgaria \\ ${ }^{3}$ Department of Information Technology, Techno India College of Technology, India \\ ${ }^{4}$ Department of Electronics and Electrical Communications Engineering, Faculty of Engineering, Tanta University, Egypt
}

\begin{abstract}
The article illustrates the results of a scientific research of heart rate variability (HRV) of a group of patients with type2 diabetes. This analysis is based on digital electrocardiograms and characterized as a non-invasive and effective tool to reflect the autonomic nervous system regulation of the heart. Heart rate variability is used to diagnose and estimate the alterations in heart rate by measuring the variation of the time intervals between two consecutive heart beats (RR) intervals. During the study RR time series of both healthy and type 2 diabetic patients are extracted from electrocardiograms through the "Polar Advantage Interface" device. The proposed study employed three methods for nonlinear analysis of the RR time series: Detrended Fluctuation Analysis $(\alpha 1, \mathrm{p}<0.0001 ; \alpha 2, \mathrm{p} \approx 0.06 ; \alpha$ all, $\mathrm{p}<0.05)$, Poincaré plot (SD1, $\mathrm{p}<0.05$; SD2, $\mathrm{p}<0.0001$; SD1/SD2, $\mathrm{p}<0.0001)$ and Rescaled adjusted range Statistics plot $(\mathrm{H}, \mathrm{p}<0.0001)$. The results from the experiments conclude that these methods are suitable for diagnostic, forecast and prevention of the pathological statuses.
\end{abstract}

\section{Introduction}

Diabetes mellitus (DM) is a chronic, lifelong condition that affects the body's ability to metabolize the carbohydrates in order to release. Diabetes is a very common disease. Currently, there are about 366 million diabetic patients, which expected to reach 522 million by the year 2030 [1]. DM can cause cardiovascular autonomic neuropathy that is associated with increased cardiovascular mortality [2-4]. Therefore, the diagnosis and monitoring of diabetes is of a great significance. Diabetes can be easily diagnosed by measuring the HRV, which is a physiological phenomenon of variation in the time interval between heartbeats [5]. ECG is a non-invasive device that used to assess the HRV by measuring the time between heartbeats. In 1996, the European Society of Cardiology and North American Society of Pacing and Electrophysiology provided recommendations on clinical usage of the HRV for the evaluation of the risk for cardiology disease such as myocardial infarction (heart attacks) and sudden cardiac death [6]. The HRV measurements are nonlinear and non-stationary [7]. Through implementation of the conventional (linear) mathematical methods, part of the important characteristics of the signal dynamics is lost. The research, development and implementation of a new nonlinear mathematical schemes based on the fractal theory will allow to better estimate the cause for HRV fluctuations. These nonlinear methods are based on the relationship between the HRV's characteristics, such as the self-similarity, scalability, fractal dimension, long-range dependence $[8,9]$, which are quite similar to those of the fractal geometry. The cardiac data generated by using nonlinear methods can provide detailed information about the physiological status of the patients as well as develop break-through knowledge regarding the diagnostics, prognosis and prevention of cardiovascular disease pathology. Recently, the nonlinear analysis of the cardiology data becomes a new scientific trend that presents a novel concept for the heart activity dynamics.
The main objective of this article is to evaluate the impact of Type 2 Diabetic mellitus (T2DM) on HRV by using three graphical nonlinear methods: Detrended Fluctuation Analysis, Poincaré plot and Rescaled adjusted range Statistics plot.

\section{Materials and methods}

The study group consisted of 25 patients (mean age $52.8 \pm 5.1$ years) diagnosed with T2DM. The control group consisted of 22 healthy subjects (mean age $50.3 \pm 4.5$ years). All patients are subject to a 5 minute record of the RR time intervals. The RR time series are extracted from the ECG signals using the "Polar Advantage Interface" device (Polar Electro, Finland). The current work developed software for the HRV analysis based on the mentioned graphical nonlinear methods [16].

\section{Detrended fluctuation analysis}

The DFA [10] is a technique for detecting correlations in time series. These functions are able to estimate several scaling exponents from the $\mathrm{RR}$ time series being analyzed. The scaling exponents characterize short or long-term fluctuations.

The main steps of the DFA algorithm are as follows [10]:

Correspondence to: Mitko Gospodinov, Institute of Systems Engineering and Robotics, Bulgarian Academy of Sciences, Bulgaria, E-mail: mitgo@abv.bg

Key words: electrocardiogram (ECG), heart rate variability (HRV), nonlinear analysis, detrended fluctuation analysis (DFA), poincaré plot, rescaled adjusted range statistics plot $(R / S)$

Received: February 23, 2016; Accepted: March 23, 2016; Published: March 26 2016 
1. The RR interval time series is integrated using:

$$
y(K)=\sum_{j=1}^{k}\left(R R_{j}-\overline{R R}\right), k=1, \ldots, N
$$

Where:

- $\quad y(k)$ is the $k^{\text {th }}$ value of the integrated series;

- $\quad \mathrm{RR}_{\mathrm{j}}$ is the $\mathrm{j}^{\text {th }}$ inter beat interval;

$\bar{R}$ is the average inter beat interval over the entire series.

2. The integrated time series is divided into boxes of equal length $\mathrm{n}$.

3. In each box of length $\mathrm{n}$, a least square line is fitted to the $\mathrm{RR}$ interval data and $\mathrm{y}_{\mathrm{n}}(\mathrm{k})$ denotes the regression lines.

4. The integrated series $y(k)$ is detrended by subtracting the local trend in each box. The root-mean-square fluctuation of this integrated and detrended series is calculated by:

$$
F(n)=\sqrt{1 / N \sum_{k=1}^{N}\left(y(k)-y_{n}(k)\right)^{2}}
$$

Where: $F(n)$ is a fluctuation function of box size $n$.

5. This procedure is repeated for different time scales. The relationship on a double-log graph between fluctuations $\mathrm{F}(\mathrm{n})$ and the time scales $\mathrm{n}$ can be approximately evaluated by a linear model that provides the scaling exponent $\alpha$ given by:

$$
F(n) \approx n^{\alpha}
$$

The parameter a depends on the correlation properties of the signal. By changing the parameter $\mathrm{n}$ can be studied how to change the fluctuations of the signal. Linear behavior of the dependence $F(n)$ is an indicator of the presence of a scalable behavior of the signal. The value of the parameter $\alpha$ is determined from the slope of the straight line. For uncorrelated signals, the value of this parameter is within the range $(0$, 0.5 ), where $\alpha>0.5$ indicates the presence of correlation. While, for

$$
\alpha= \begin{cases}1 & \text { signal is } 1 / \mathrm{f} \text { (noise) } \\ 1.5 & \text { Brownian motion }\end{cases}
$$

In the case of RR time series, the DFA shows typically two ranges of scale invariance, which are quantified by two separate scaling exponents, $\alpha_{1}$ and $\alpha_{2,}$ reflecting the short-term and long-term correlation [11].

\section{Poincaré plot}

The Poincaré plot analysis is a graphical nonlinear method to assess the dynamic of HRV. This method provides summary information as well as detailed beat-to-beat information on the behavior of the heart. It is a graphical representation of temporal correlations within the RR intervals derived from the ECG signal. The Poincaré plot is a return maps or scatter plots, where each RR interval from time series $\mathrm{RR}=$ $\{R R 1, R R 2, \ldots, R R n, R R n+1\}$ is plotted against the next RR interval. The Poincaré plot parameters used in this paper are SD1, SD2 and SD1/SD2 ratio. SD1 is the standard deviation of projection of the Poincare plot on the line perpendicular to the line of identify. While, the SD2 is defined as the standard deviation of the projection of the Poincaré plot on the line of identify $(\mathrm{y}=\mathrm{x})$. These parameters can be defined by following equations $[12,13]$ :

$$
\begin{aligned}
& \begin{array}{l}
x=\{x 1, x 2, \ldots, x n\}=\{R R 1, R R 2, \ldots, R R n\} \\
\quad y=\{y 1, y 2, \ldots, y n\}=\{R R 2, R R 3, \ldots, R R n+1\}
\end{array} \\
& S D 1=\sqrt{\operatorname{var}\left(d_{1}\right)} ; S D 2=\sqrt{\operatorname{var}\left(d_{2}\right)} ; \text { Ratio }=\frac{S D 1}{S D 2} \\
& \text { Where: }
\end{aligned}
$$

$-\mathrm{i}=1,2,3, \ldots, \mathrm{n}$ and $n$ is the number of points in the Poincare plot; - $\operatorname{var}(\mathrm{d})$ is the variance of $\mathrm{d}$;

$$
\mathrm{d}_{1}=\frac{\mathrm{x}-\mathrm{y}}{\sqrt{2}} ; \mathrm{d}_{2}=\frac{\mathrm{x}+\mathrm{y}}{\sqrt{2}} \text {. }
$$

The parameter SD1 has been correlated with high frequency power, while SD2 has been correlated with both low and high frequency powers. The ratio SD1/SD2 is associated with the randomness of the HRV signal. Thus, this ratio is a measure of the randomness in HRV time series. It has been suggested that the ratio SD1/SD2 has the strongest association with mortality in adults.

\section{Rescaled adjusted range Statistics plot}

The rescaled range is a statistical measure of the variability of a time series introduced by British hydrologist Harold Hurst [14]. Closely associated with R/S method is the Hurst exponent. This exponent is used to evaluate the presence or absence of the long-range dependence and its degree in a time series. Based on the Hurst exponent value, the following classifications of the time series can be realized:

- $\quad \mathrm{H}=0.5$ indicates a random series;

$0<\mathrm{H}<0.5$ indicates that the data in the signal are anticorrelated;

- $\quad 0.5<\mathrm{H}<1$ indicates that the data in the signal are long-range correlated. law:

The R/S for the time series $\mathrm{X}(\mathrm{n})$ is asymptotically given by a power

$$
R(n) / S(n) \neq n^{H}
$$

Where:

- $\quad \mathrm{R}(\mathrm{n})$ is the range which is the difference between the minimum and maximum accumulated values;

- $\quad \mathrm{S}(\mathrm{n})$ is the standard deviation estimated from the observed data $\mathrm{X}(\mathrm{n})$;

- $\quad \mathrm{H}$ is the Hurst exponent.

To estimate the Hurst exponent, the plot of $R(n) / S(n)$ versus $n$ in $\log$-log axes is done. The slope of the regression line approximates the Hurst exponent [15]. This exponent characterization of the fractal structure of the self-similar signals.

\section{Statistical analysis}

The results were presented as mean \pm standard deviation (SD). Differences between the parameters of the groups were tested by the unpaired $t$ test. A p-value of $<0.05$ was regarded as statistically significant. The derived HRV indices were statistically tested using receiver operating characteristics (ROC). Statistical analysis was performed using the IBM SPSS Statistics, Version 23 (IBM Corp., USA). 


\section{Results and discussion}

The described mathematical methods are implemented in the form of specialized software for the calculation and assessment of HRV parameters. The analyzed data from the medical study of patients were combined into two groups of signals: for 25 patients diagnosed with T2DM and 22 normal subjects.

Figure 1 illustrates the main software menu for each patient. The menu shows distribution of the QRS complexes (combination of three of the graphical deflections seen on the ECG), RR intervals and results of time-domain analysis (the values of investigated parameters, RR and HR histograms). It is available to opt one of the following analyses: time-domain, frequency-domain, nonlinear and wavelet analysis from this page [16]. The current work illustrated the results of the nonlinear analysis.

Figure 2(left) and Figure 2(right) demonstrated the RR signals of normal and T2DMpatients. The fluctuations of the heart-beat time series are larger in healthy subject compared to T2DM patients.

Figure 3(left) and Figure 3(right) showed the values of scaling exponents and the slope of the line $\mathrm{F}(\mathrm{n})$ on double logarithmic plot obtained by using the DFA method for the investigated signals. The results showed difference between values of the scaling exponents $\alpha_{1}$ and $\alpha_{2}$ for T2DM patient and healthy one.

The results of the Poincaré plot analysis of RR time series for healthy subject and T2DM patients are displayed in Figure 4 (left) and Figure 4 (right). The Poincaré plot for healthy subject is a cloud of points in shape of an ellipse. On the other hand, points for patient with T2DM are a cloud of points in shape of a circle. The geometry of these plots has been shown to distinguish between healthy and unhealthy subjects.

The results of the R/S method applied to the studied signals to determine the value of the Hurst exponent are shown in Figure 5 (left) and Figure 5 (right). The obtained results demonstrated that the RR time series are correlated, i.e., they are fractal time series.

Diabetes causes cardiovascular autonomic neuropathy that affects the HRV. The main finding of the present study concluded that the HRV significant is differed between the T2DM patients and of healthy subjects. The effects of T2DM on different HRV parameters are evaluated using data from 25 patients with T2DM and 22 healthy subjects.

Various literatures were concerned with the study of the cardiac signal analysis and measurements [16-21]. Meanwhile, from the

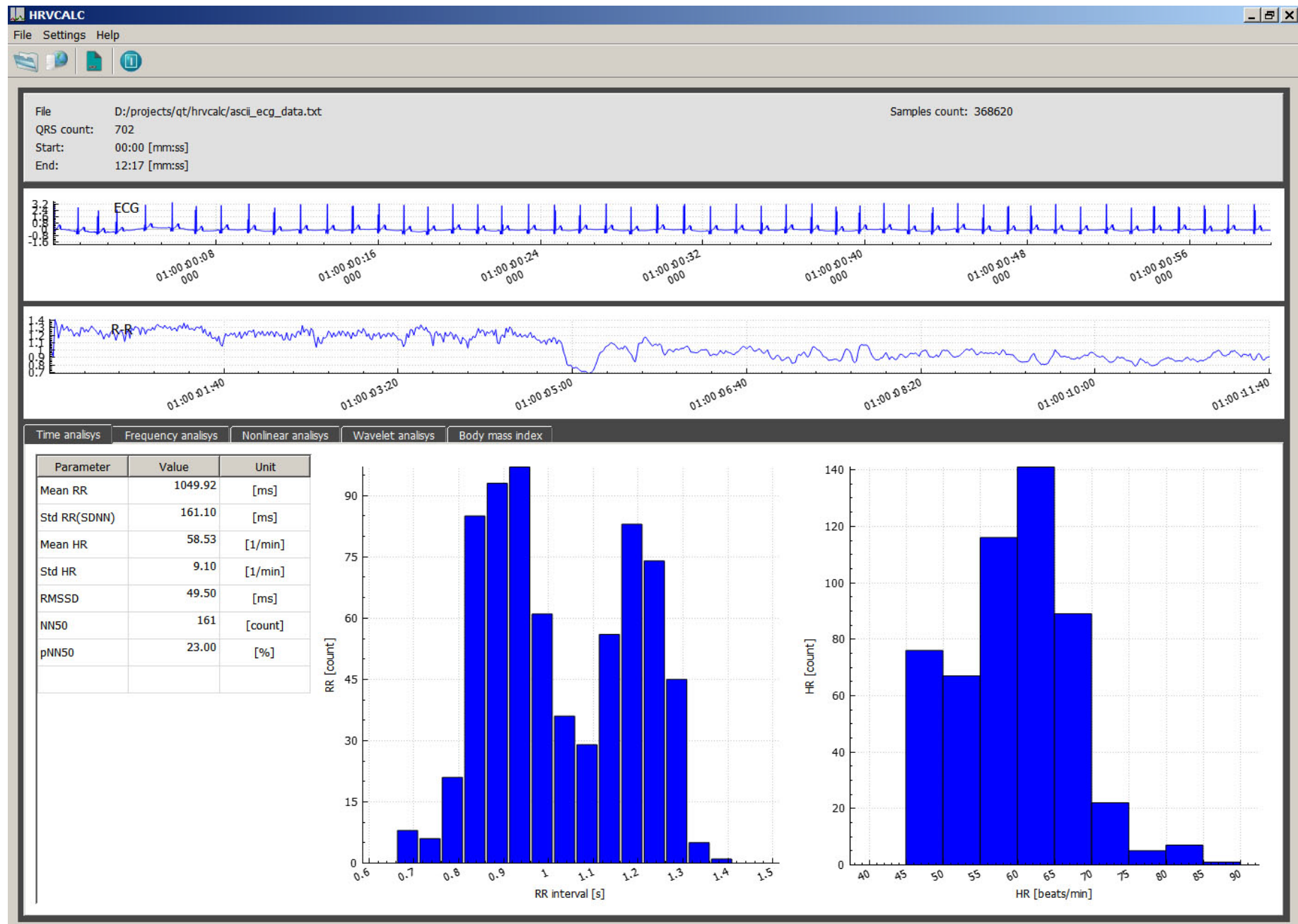

Figure 1. Proposed software main menu for HRV analysis [16]. 

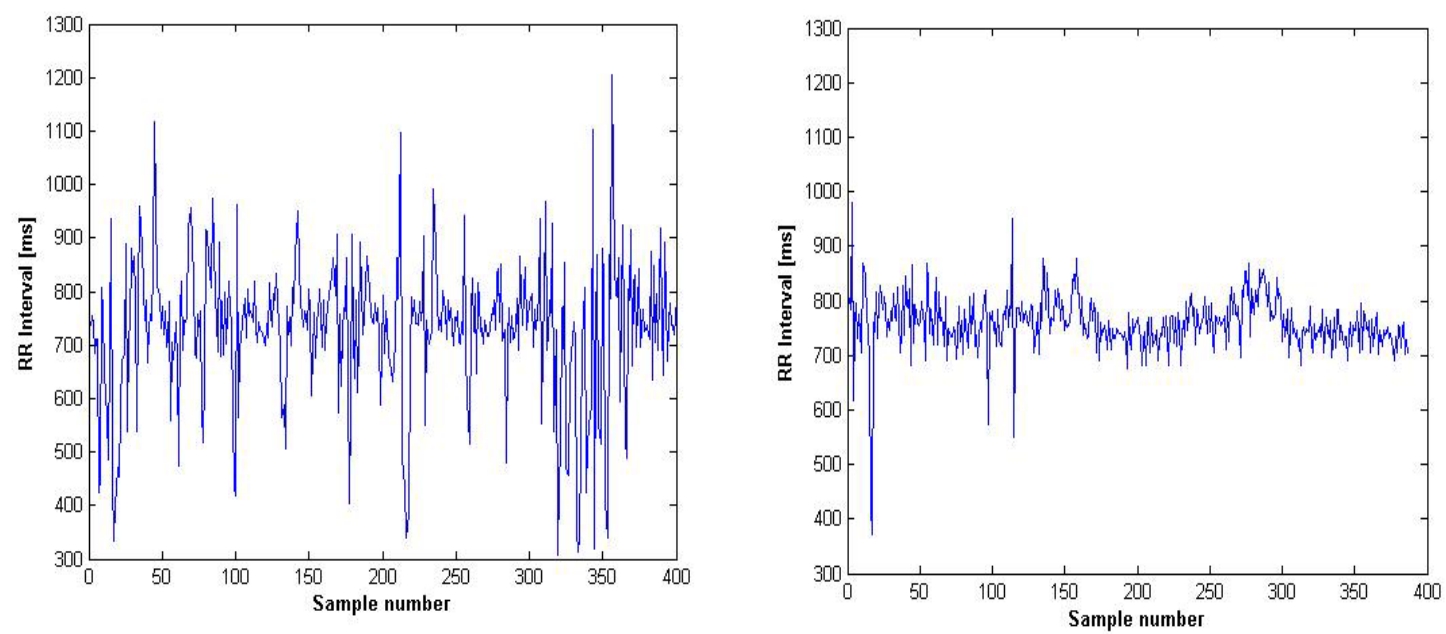

Figure 2. RR-interval signal of a healthy subject (left) and diabetic patient (right).
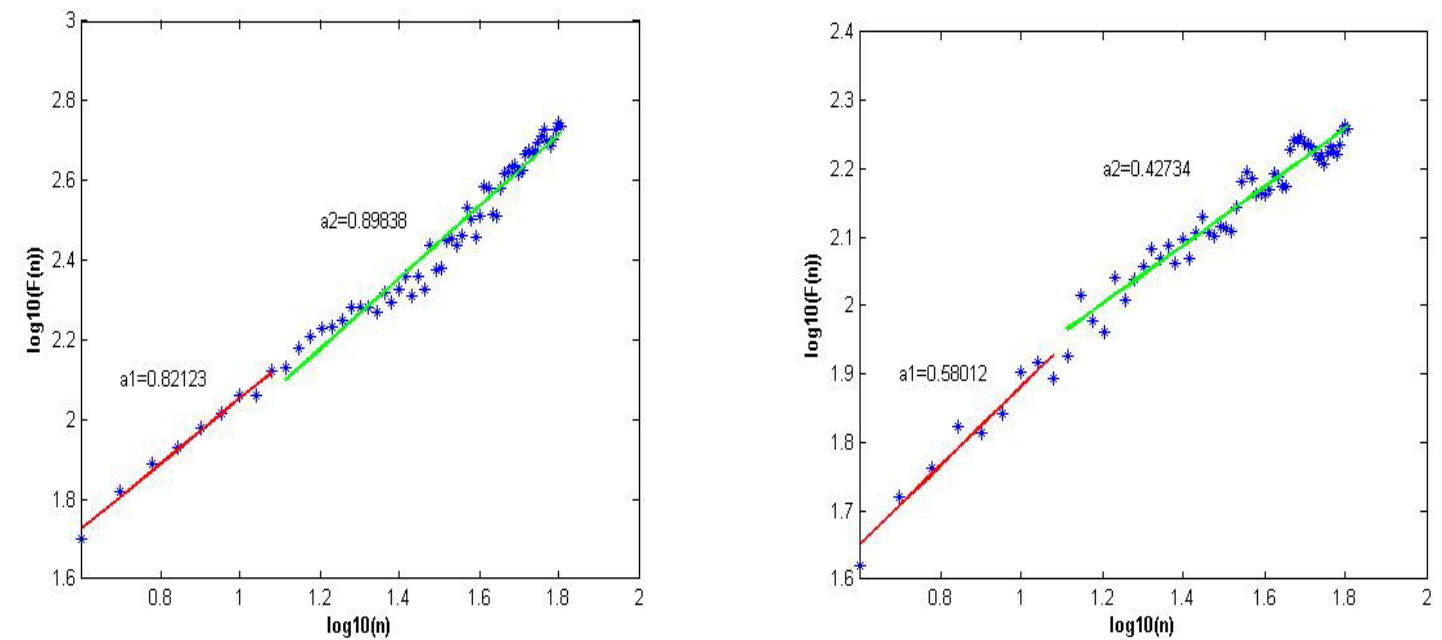

Figure 3. DFA analysis of a healthy subject (left) and diabetic patient (right).
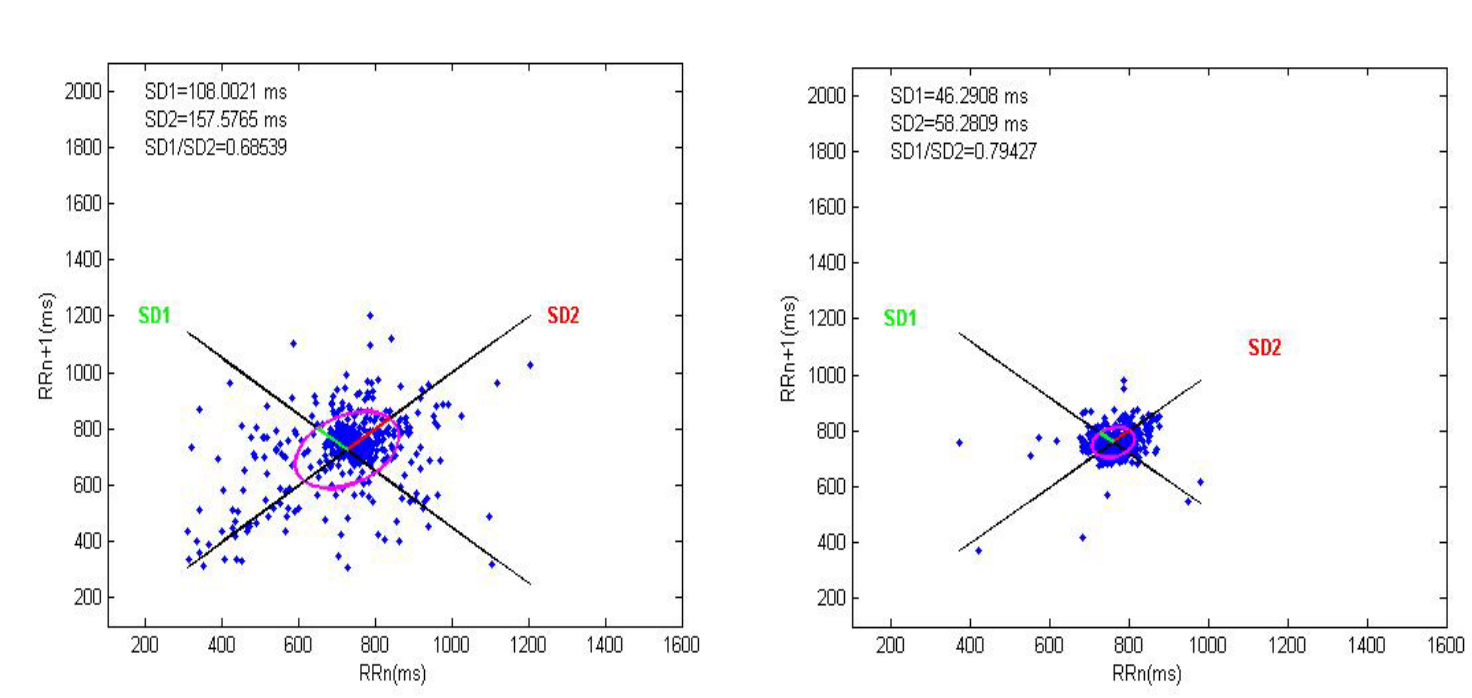

Figure 4. Poincaré plot analysis of a healthy subject (left) and diabetic patient (right). 

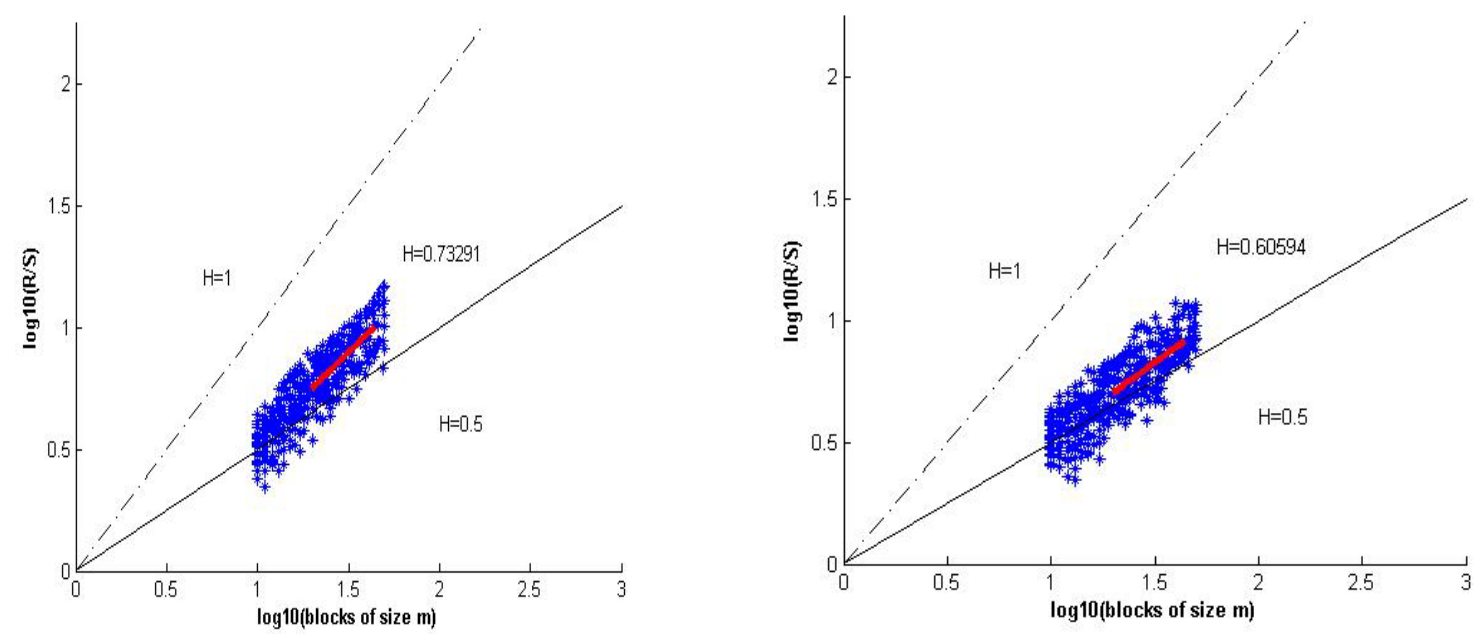

Figure 5. R/S analysis of a healthy subject (left) and diabetic patient (right).

Table 1. Parameters for DM type 2 and healthy subjects.

\begin{tabular}{|c|c|c|}
\hline Parameter & Diabetic Patients & Healthy Subjects \\
\hline$\alpha_{1}$ (DFA) & $0,594 \pm 0,173$ & $0,792 \pm 0,075$ \\
\hline$\alpha_{2}$ (DFA) & $0,769 \pm 0,184$ & $0,848 \pm 0,057$ \\
\hline$\alpha_{\text {all }}$ (DFA) & $0,739 \pm 0,137$ & $0,825 \pm 0,033$ \\
\hline SD1[ms] (Poincaré plot) & $53,512 \pm 11,719$ & $66,357 \pm 16,084$ \\
\hline SD2[ms] (Poincaré plot) & $62,580 \pm 19.135$ & $<3,244 \pm 15,774$ \\
\hline SD1/SD2(Poincaré plot) & $0,925 \pm 0,177$ & 0.0597 \\
\hline Hurst (R/S) & $0,609 \pm 0,110$ & $<0.726 \pm 0,062$ \\
\hline
\end{tabular}

Table 2. Analysis of HRV parameters using ROC.

\begin{tabular}{|c|c|c|c|c|}
\hline Parameter & Sensitivity (\%) & Specificity (\%) & AUC & $\begin{array}{l}\text { 95\% Confidence Interval } \\
\text { (Lower Bound-Upper Bound) }\end{array}$ \\
\hline$\alpha_{1}(\mathrm{DFA})$ & 72.87 & 55.24 & 0.724 & $(0.510-0.938)$ \\
\hline$\alpha_{2}(\mathrm{DFA})$ & 60.21 & 59.61 & 0.371 & $(0.138-0.604)$ \\
\hline$\alpha_{\text {all }}(\mathrm{DFA})$ & 77.68 & 46.28 & 0.776 & $(0.582-0.971)$ \\
\hline SD1[ms] (Poincaré plot) & 73.50 & 42.30 & 0.829 & $(0.655-1.000)$ \\
\hline SD2[ms] (Poincaré plot) & 70.64 & 42.82 & 0.752 & $(0.547-0.958)$ \\
\hline SD1/SD2(Poincaré plot) & 83.33 & 52.86 & 0.962 & $(0.891-1.000)$ \\
\hline Hurst $(\mathrm{R} / \mathrm{S})$ & 67.63 & 51.80 & 0.767 & $(0.567-0.966)$ \\
\hline
\end{tabular}

current study the following significant discussion can be given based on the experimental results obtained concerned with the HRV nonlinear analysis.

The DFA method is used to quantity the fractal scaling properties of RR time signals. The values of fractal scaling exponents for T2DM patients are lower than for the healthy one.

The Poincaré plot parameters are directly related to the physiology of the heart. The parameter SD1 is the reflection of short term variability of heart rate and parameter SD2 is the measure of long-term variability. The values of these two parameters are lesser in normal subjects than for the T2DM patients.

The values of the Hurst exponents of T2DM patients are smaller than these of healthy subjects. The scientific researches [7,9] determined the Hurst exponent decrease of physical, mentally activity and cardio disease. The investigation of dependence between the value of the Hurst exponent and the cardiology status could be used for diagnosis and prognosis of cardio disease.
The values of the investigated parameters for DM type 2 and healthy subjects are reported in Table 1 . The derived HRV indices were statistically tested using ROC. The ability of ROC to discriminate between diabetic and healthy patient population was determined by studying the area under the ROC curve (AUC), sensitivity and specificity (Table 2).

\section{Conclusion}

The HRV analysis is a popular noninvasive tool for assessing the activities of the cardiovascular autonomic dysfunction. Three nonlinear methods to identify the parameters associated with HRV were applied. The values of these parameters were different for both T2DM and healthy subjects. These nonlinear methods are quite useful for diagnosing the disease at an early stage as well or determining the extent. The developed application software for the illustrated above methods, designed for nonlinear analysis of ECG signals, could be utilized by physicians as an additional mathematical tool for presentation of pathological status of patients. 


\section{References}

1. Global Diabetes Plan 2011-2021. International Diabetes Federation, Belgium.

2. American Diabetes Association; National Heart, Lung and Blood Institute; Juvenile Diabetes Foundation International; National Institute of Diabetes and Kidney Disease; American Heart Association. Diabetes mellitus: a major risk factor for cardiovascular disease, 1999, Circulation, 100: 1132-1133.

3. Kudat H, Akkaya V, Sozen AB, Salman S, Demirel S, et al. (2006) Heart rate variability in diabetes patients. J Int Med Res 34: 291-296. [Crossref]

4. Tarvainen MP, Cornforth DJ, Kuoppa P, Lipponen JA, Jelinek HF (2013) Complexity of heart rate variability in type 2 diabetes - effect of hyperglycemia. Conf Proc IEEE Eng Med Biol Soc 2013: 5558-5561. [Crossref]

5. Mirza M, Lakshmi ANK (2012) A comparative study of Heart Rate Variability in diabetic subjects and normal subjects. International Journal of Biomedical and Advance Research 3: 640-644.

6. Task Force of the European Society of Cardiology and the North American Society of Pacing and Electrophysiology, 1996. Heart rate variability: standards of measurement, physiological interpretation, and clinical use. Circulation 93: 1043-1065.

7. Ivanov PC, Amaral LA, Goldberger AL, Havlin S, Rosenblum MG, et al. (1999) Multifractality in human heartbeat dynamics. Nature 399: 461-465. [Crossref]

8. Smith RL, Wathen ER, Abaci PC, Bergen NHV, Law IH, et al. (2009) Analyzing Heart Rate Variability in Infants Using Non-Linear Poincare Techniques. Computer in Cardiology 36: 673-876.

9. Stanley HE, Amaral LA, Goldberger AL, Havlin S, Ivanov PCh, et al. (1999) Statistical physics and physiology: monofractal and multifractal approaches. Physica A 270: 309 324. [Crossref]

10. Peng CK, Havlin S, Stanley HE, Goldberger AL (1995) Quantification of scaling exponents and crossover phenomena in nonstationary heartbeat time series. Chaos 5 82-87. [Crossref]

11. Baumert M, Javorka M, Seeck A, Faber R, Sanders P, et al. (2012) Multiscale entropy and detrended fluctuation analysis of QT interval and heart rate variability during normal pregnancy. Comput Biol Med 42: 347-352. [Crossref]
12. Rhaman Md, Karim AHM, Hasan M, Sultana J (2013) Successive RR Interval Analysis of PVC with Sinus Rhythm Using Fractal Dimension, Poincare Plot and Sample Entropy Method. I.J Image, Graphics and Signal Processing 2: 17-24.

13. Hurst HE, Black RP, Sinaika YM (1965) Long-term Storage in Reservoirs: An experimental Stud, Constable, London.

14. Gospodinov M, Gospodinova E (2005) The graphical methods for estimating Hurst parameter of self-similar network traffic. International Conference on Computer Systems and Technologies pp. IIIB.19-1-IIIB.19-6.

15. Gospodinova E, Gospodinov M, Georgieva-Tsaneva G, Cheshmedjiev K (2015) Spectral analysis of heart rate variability. International Conference AUTOMATICS AND INFORMATICS, Bulgaria, Sofia, pp 95-98.

16. Dey N, Das A, Chaudhuri SS (2012) Wavelet Based Normal and Abnormal Heart Sound Identification Using Spectrogram Analysis. International Journal of Computer Science \& Engineering Technology 3.

17. Dey N, Samanta S, Yang SH, Chaudhri SS, Das A (2013) Optimisation of Scaling Factors in Electrocardiogram Signal Watermarking using Cuckoo Search. International Journal of Bio-Inspired Computation 5: 315-326.

18. Mukherjee A, Dey G, Dey M, Dey N (2014) Web-based Intelligent EEG signa Authentication and Tamper Detection System for Secure Telemonitoring. Published by Brain-Computer Interfaces: Current Trends and Applications by Springer-Verlag, Germany, 2014

19. Nandi S, Roy S, Dansana J, Karaa W, Ray R, et al. (2014) Cellular Automata based Encrypted ECG-hash Code Generation: An Application in Inter-human Biometric Authentication System. International Journal of Computer Network and Information Security 11: 1-12.

20. Gospodinova E, Gospodinov M, Domuschiev I, Nilianjan Dey, Ashour AS, et al (2015) Analysis of Heart Rate Variability by Applying Nonlinear Methods with Different Approaches for Graphical Representation of Results. International Journal of Advanced Computer Science and Applications 6: 38-45.

21. Acharjee S, Dey N, Samanta S, Das D, Roy R, et al. ECG Signal compression using Ant Weight Lifting Algorithm for Tele-monitoring. Journal of Medical Imaging and Health Informatics [In - press]

Copyright: $@ 2016$ Gospodinov M. This is an open-access article distributed under the terms of the Creative Commons Attribution License, which permits unrestricted use, distribution, and reproduction in any medium, provided the original author and source are credited. 